PAPER FOR MOLECULAR PHYSICS, SPECIAL ISSUE ON "ATOMS, MOLECULES, AND CLUSTERS IN MOTION"

\title{
Ab-initio modelling of molecular hydrogen rotation in the outside of carbon nanotubes
}

\author{
María Pilar de Lara-Castells ${ }^{\mathrm{a}}$ and Alexander O. Mitrushchenkov ${ }^{\mathrm{b}}$ \\ ${ }^{a}$ Instituto de Física Fundamental (C.S.I.C.), Serrano 123, E-28006, Madrid, Spain; \\ b Université Paris-Est, Laboratoire Modélisation et Simulation Multi Echelle, MSME UMR \\ 8208, CNRS, UPEC, UPEM, F-77454, Marne la Vallée, France
}

\section{ARTICLE HISTORY}

Compiled November 18, 2018

\begin{abstract}
A first-principle modelling of hydrogen molecular rotation in the outside of carbon nanotubes is presented. Density functional theory (DFT)-based symmetry-adapted perturbation theory (SAPT) is first applied to analyze the influence of the rotation on the dispersion and dispersionless $\mathrm{H}_{2}$-nanotube interaction for both sub- and nanometer-sized tubes. An adsorbate three-dimensional wave-function treatment is then applied to calculate the molecular energy levels of the rotating hydrogen molecule. As a key difference with the $\mathrm{H}_{2}$ located inside the tubes, the SAPT-based analysis indicates a marked influence of a nanotube curvature-induced dipole on the angular-dependent balance of exchange-repulsion, electrostatic, and dispersion contributions for narrow nanotubes. As a result, the landscape of molecular energy levels depends strongly on the diameter of the porous material. In addition, an effective one-dimensional model is proposed to account for the nuclear motion, reproducing full-dimensional approach within less than $1 \%$.
\end{abstract}

\section{KEYWORDS}

carbon nanotubes; quantum confinement; molecular hydrogen rotation; curvature-dependent dipole

\section{Introduction}

Extraordinary properties such as chemical stability, electric conductivity, non-toxicity, high surface area, and precisely tuned pores of carbon nanotubes (CNTs) make them relevant nanomaterials for many modern applications ranging from hydrogen storage [1] to cancer diagnosis [2]. Remarkably, both experimental and theoretical evidences for hydrogen solid-like packing have been very recently reported [3, 4]. Accordingly, the research on methods to produce CNTs has been intensified over the last few years, including synthesis techniques from waste plastics [5] with hydrogen co-production [6].

On a fundamental level, the analysis of the role of quantum nuclear effects (QNEs) in the low-temperature motion of light molecules and atoms such as hydrogen and helium $[7,8]$ in carbon nanotubes has attracted much attention. The impact of QNEs has been manifested in theoretical studies characterizing the (superfluid or crystalline) phase of parahydrogen molecules inside carbon nanotubes at zero $[9,10]$ and ultra-low

Corresponding Authors. Email: Pilar.deLara.Castells@csic.es; Email: Alexander.Mitrushchenkov@u-pem.fr 
temperatures [11]. A quantum-induced reversed trend in $\mathrm{H}_{2}$ and $\mathrm{D}_{2}$ diffusion rates upon lowering the temperature has also been experimentally detected [12] and theoretically confirmed [13]. Moreover, QNEs effects upon confining molecular hydrogen in the outside of aligned carbon nanotubes [14] and carbon nanohorns [15] have been manifested using high-resolution neutron spectroscopy. This technique has allowed to characterize accurately the energy levels of molecular hydrogen and, in particular, to provide values of the $m$-splittings resulting from the anisotropy of the $\mathrm{H}_{2}$-nanotube interaction [14].

On the theoretical side, the development of computational tools capable of providing precise values of energy levels, including $m$-splitting values, is thus necessary, beyond the treatment of hydrogen molecules as structureless, spherically symmetric, particles [4]. Very recently, we have developed an effective three-dimensional approach capable of providing them for molecular hydrogen rotating inside carbon nanotubes [16]. Our method is based on a potential model for the van der Waals (vdW)-dominated $\mathrm{H}_{2}-$ nanotube interaction relying on ab-initio DFT-based symmetry-adapted perturbation theory (SAPT) to derive the parameters of an effective pairwise potential model $[4,8]$. In fact, ab-initio intermolecular interaction theory has recently allowed an accurate description of dispersion-dominated interactions of molecules with carbon nanostructures $[7,8,17-24]$. Recent options include schemes combining second-order MöllerPlesset perturbation theory with the coupled-cluster approach $[17,18]$, as well as a non-periodic implementation of the incremental method [25] applied at coupled-cluster level [26-30] with periodic calculations employing dispersionless density functional theory $[29,31-33]$.

Our previous study [16] was focused on molecular hydrogen rotating inside carbon nanotubes and revealed the key role of the anisotropy of the $\mathrm{H}_{2}$-interaction and the diameter of the nanotube in the values of the $m$-splittings, and, more precisely, in the energy barriers from parallel to perpendicular $\mathrm{H}_{2}$ orientations with respect to the tube long axis. For CNTs of various size, molecular hydrogen showed a preferential orientation in a parallel arrangement. This preferential orientation stemmed from a counterbalance of dispersionless (mainly exchange-repulsion) and dispersion energy contributions, favoring parallel and orthogonal configurations, respectively. Upon increasing the nanotube diameter, the dispersion energy contribution becomes more relevant, resulting in reduced energy barriers between different orientations. In the limiting case of $\mathrm{H}_{2}$ adsorbed on planar graphene, our earliest study [19] showed consistently small $m$-splitting values but also a preferential orthogonal orientation with respect to the graphene plane.

Previous findings for $\mathrm{H}_{2}$ rotating either inside CNTs [16] or onto graphene sheets [19] raise the question of whether the preferential $\mathrm{H}_{2}$ orientation is parallel or orthogonal when located in the outside of CNTs or, more generally, how much the $\mathrm{H}_{2}$-CNT interaction is influenced by the $\mathrm{H}_{2}$ rotation and the CNT diameter, and how these features are reflected in the molecular energy levels. In this context, the diameterdependent dipole oriented towards the outside of the nanotube [34] might affect very significantly the partitioning of the $\mathrm{H}_{2}$-nanotube interaction into the different (i.e., exchange-repulsion, electrostatic, and dispersion) contributions and, then, the balance determining the anisotropy of the interaction and the structure of the molecular energy levels.

To answer these questions, a SAPT-based analysis of the interaction is first presented for CNTs with diameter below and above one nanometer. Second, our adsorbate three-dimensional wave function method [16] is adapted to calculate the associated energy levels. Finally, an effective one-dimensional model is proposed, reproducing full- 
dimensional approach values to within $1 \%$. The next section is dedicated to a brief description of the computational method. Results are reported in Section 3. Finally, the main conclusions are provided in Section 4.

\section{Method}

\subsection{SAPT-based decomposition of the adsorbate-nanotube interaction}

A carbon nanotube (CNT) is typically characterized by the so called helicity index $(m, n)$ (see, e.g., Ref. 35). We have considered $(5,5)$ and $(10,10)$ CNTs as examples of tubes with diameter below and above one nanometer (6.74 and $13.56 \AA$, respectively). To analyze the $\mathrm{H}_{2} / \mathrm{CNT}$ interaction, the SAPT(DFT) method [36, 37] has been applied as implemented within the MOLPRO electronic structure package [38] including density fitting (DF) [39]. This method allows to decompose the $\mathrm{H}_{2}$-nanotube interaction energy as a function of the angular orientation of the molecular adsorbate. Specifically, the total interaction energy is written as a sum of first- and second-order interaction terms, namely first-order electrostatic $E_{\text {elec }}$ and exchange $E_{\text {exch }}$, second-order induction $E_{\text {ind }}$ and dispersion $\mathrm{E}_{\text {disp }}$ terms, along with their respective exchange corrections ( $\mathrm{E}_{\text {exch-ind }}$ and $\left.\mathrm{E}_{\text {exch-disp }}\right)$. The dispersion and exchange-dispersion SAPT(DFT) contributions were summed to provide the total exchange-repulsion part, as were the induction and exchange-induction contributions into the total induction part.

We followed the same computational setup reported in previous works $[4,8,16]$, using the Perdew-Burke-Ernzerhof (PBE) density functional [40] and a modified version of the augmented polarized correlation-consistent triple-zeta basis [41] (aug-cc-pVTZ) for the nanotube carbon atoms as well as for the adsorbate hydrogen atoms. For the specific case of the nanotube $\mathrm{CNT}(10,10)$, the cc-pVTZ basis set for the nanotube carbon atoms was employed instead. The DF of Coulomb and exchange integrals employs the auxiliary basis set developed for the aug-cc-pVTZ basis by Weigend [42], while the aug-cc-pVTZ/MP2Fit basis [43] was used for fits of the integrals containing virtual orbitals. The exchange-correlation PBE potential was asymptotically corrected [44] using the ionization potential (IP) value reported in the NIST Chemistry Web Book for molecular hydrogen [45] while the IP value for the nanotube was estimated using the DFT PBE0 approach [46]. The length of these "short" nanotubes used to parametrize $\mathrm{H}_{2}$-CNT interactions, was of 4 carbon plane sections, or $3.69 \AA$. Thus, the total number of carbon atoms was 40 for $\operatorname{CNT}(5,5)$ and 80 for $\operatorname{CNT}(10,10)$. This number was limited by computationally demanding SAPT (DFT) calculations.

\subsection{Calculation of molecular energy levels}

To calculate the energy levels of the $\mathrm{H}_{2}$ molecule rotating in the outside of CNTs, we followed the same approach described in our previous work [16], which is briefly outlined here. Essentially, the six-dimensional (6D) problem is reduced to four dimensions by first removing the overall translation of $\mathrm{H}_{2}$ along the tube and keeping the $\mathrm{H}-\mathrm{H}$ distance $\left(r_{0}\right)$ fixed. This approximation is justified because the vibrational motion of $\mathrm{H}_{2}$ has a different energetic scale than the nuclear motion in which we are interested. Finally, the overall rotation of $\mathrm{H}_{2}$ as a whole around CNT is separated (the polar coordinate $\Phi$, see Ref. 16), being represented by the overall rotation quantum number $\Lambda$. Hence, we are left with three relevant coordinates: the $\mathrm{H}_{2}$ center-of-mass polar radius with respect to the center of the nanotube, $R$, and two angles describing 


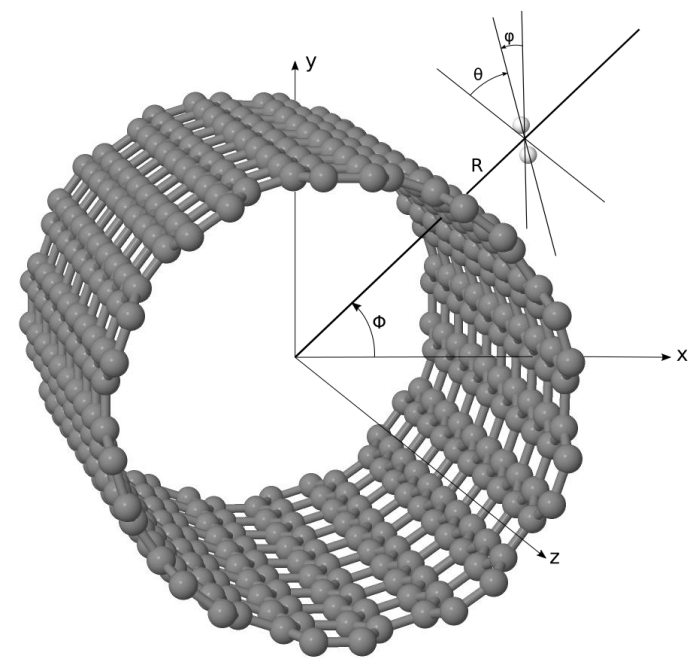

Figure 1. Figure illustrating the atomic structural model employed for the $\mathrm{H}_{2} / \mathrm{CNT}$ system. Gray spheres represent carbon atoms while white spheres stand for a single $\mathrm{H}_{2}$ molecule rotating in the outside of a carbon nanotube CNT. $R$ is the distance from the $\mathrm{H}_{2}$ center-of-mass to the nanotube axis while $\theta$ and $\phi$ are the angles defining the orientation of the $\mathrm{H}-\mathrm{H}$ internuclear axis with respect to the direction of the carbon nanotube.

the orientation of the $\mathrm{H}-\mathrm{H}$ axis with respect to $\mathrm{CNT}$ direction, $\theta$ and $\phi$ (see Ref. 16 for further details). As can be seen in Figure 1, $\theta$ is the angle between the $\mathrm{H}-\mathrm{H}$ and the $\mathrm{CNT}$ axes, and $\phi$ represents the polar angle of the $\mathrm{H}-\mathrm{H}$ axis with respect to the direction connecting nanotube center and the $\mathrm{H}_{2}$ center-of-mass. $Z$ axis is oriented along the CNT, as shown in Figure 1. Explicitly, the carthesian coordinates of the hydrogen atoms are given by

$$
\begin{aligned}
& x_{1,2}=R \cos \Phi \pm \frac{r}{2} \sin \theta \cos (\Phi+\phi) \\
& y_{1,2}=R \sin \Phi \pm \frac{r}{2} \sin \theta \sin (\Phi+\phi) \\
& z_{1,2}=Z \pm \frac{r}{2} \cos \theta
\end{aligned}
$$

where $Z$ represents the $Z$ coordinate of the $H_{2}$ center-of-mass. The resulting $\Lambda$ dependent three-dimensional (3D) Hamiltonian can be written as

$$
\begin{array}{r}
\hat{H}_{\Lambda}=-\frac{1}{2 M}\left\{\frac{\partial^{2}}{\partial R^{2}}-\frac{\Lambda^{2}}{R^{2}}-\frac{2 \imath \Lambda}{R^{2}} \frac{\partial}{\partial \phi}+\frac{1}{R^{2}} \frac{\partial^{2}}{\partial \phi^{2}}\right\} \\
-\frac{1}{2 \mu}\left\{\frac{1}{r_{0}^{2}} \frac{\partial}{\partial x}\left(1-x^{2}\right) \frac{\partial}{\partial x}+\frac{1}{r_{0}^{2}\left(1-x^{2}\right)} \frac{\partial^{2}}{\partial \phi^{2}}\right\}+V(R, x, \phi)
\end{array}
$$

with $x=\cos \theta, M$ as the total mass of the $\mathrm{H}_{2}$ molecule, and $\mu$ as the reduced mass associated to the relative $\mathrm{H}-\mathrm{H}$ motion (for homonuclear molecules $\mathrm{H}_{2}, M=2 m_{\mathrm{H}}, \mu=$ $\left.m_{\mathrm{H}} / 2\right)$. Finally, $V(R, x, \phi)$ is the interaction potential between the $\mathrm{H}_{2}$ molecule and the nanotube. Its treatment differs slightly from that detailed in our previous work [16]. In fact, we have found that the representation of $V(R, x, \phi)$ as a sum of independent interactions of two individual $\mathrm{H}$ atoms with the nanotube, $V(R, x, \phi)=V_{\mathrm{H}}\left(\rho_{1}\right)+$ $V_{\mathrm{H}}\left(\rho_{2}\right)$, is not accurate enough for the $\mathrm{H}_{2}$ motion outside of the nanotube. More precisely, the dispersive part can still be described by this approximation with very 
good accuracy, as one would expect for a long-range dispersive contribution. Thus, we can write

$$
V(R, x, \phi)=V_{\text {disp-less }}(R, x, \phi)+V_{\text {disp }-\mathrm{H}}\left(\rho_{1}\right)+V_{\text {disp }-\mathrm{H}}\left(\rho_{2}\right)
$$

where $\rho_{1}$ and $\rho_{2}$ stand for the radial distance of each $\mathrm{H}$ atom to the tube axis. The dispersion part is easily calculated from that corresponding to the parallel orientation as

$$
V_{\mathrm{disp}-\mathrm{H}}(\rho)=\frac{1}{2} V_{\mathrm{disp}}(\rho, \theta=0, \phi)
$$

As proposed in Ref. 4, an additive pairwise potential model (PPM) has been used to fit the ab-initio energies for the dispersive $\mathrm{H}_{2} / \mathrm{CNT}$ interaction obtained with the SAPT(DFT) method. The parameters of the PPM model were refit for the $\mathrm{H}_{2}$ motion outside of the nanotube, and are provided in Appendix A, Table A1. We have verified that this PPM model accurately (within 1\%) reproduces the dispersive interaction of $\mathrm{H}_{2}$ with both $(5,5)$ and $(10,10) \mathrm{sCNTs}$ at three different orientations, as obtained from full SAPT calculations.

The dispersionless interaction SAPT(DFT) energies were fit for different $\mathrm{H}_{2}$ orientations and nanotube sizes. It is clear that for large nanotube diameter, the interaction should approach that of $\mathrm{H}_{2}$ and graphene sheet. In terms of our coordinates this means that the interaction for $\theta=0$ (and any $\phi$ ) should be the same as for $\theta=\phi=90$. In practice, especially for smaller diameter, they are slightly different. However, we have verified that $\theta, \phi$-dependence can be accurately reproduced using the following expansion:

$$
V_{\text {disp-less }}(R, \theta, \phi)=V_{\text {disp-less }}^{00}(R)+\sin ^{2} \theta V_{\text {disp-less }}^{10}(R)+\sin ^{2} \theta \cos ^{2} \phi V_{\text {disp-less }}^{11}(R)
$$

where

$$
\begin{array}{r}
V^{00}=V(\theta=0) \\
V^{10}=V(\theta=\phi=90)-V(\theta=0) \\
V^{11}=V(\theta=90, \phi=0)-V(\theta=\phi=90)
\end{array}
$$

To describe the dependence on $R$, we have found that the following analytic expression

$$
V_{\text {disp-less }}(R)=A\left\{\exp (-\alpha R)+\exp \left(-\beta R^{2}\right)\right\}
$$

provides accurate representation in the relevant $R$ interval. The constants $A, \alpha, \beta$ depend on the angles $\theta$ and $\phi$ and are given in Appendix A, Table A2.

The dispersionless interaction is a short-range interaction, therefore the obtained expressions provide accurate estimation for CNTs of any (infinite) length. The dispersive part decays at worst as $1 / R^{6}$. To obtain the dispersion interaction potentials of $H_{2}$ (in parallel orientation) with long CNTs, we have used the PPM model described above performing explicit summations over all carbon atoms of long CNTs. In practice, we used CNTs build of 20 plane carbon sections, thus of a total length of 23.4 
$\AA$. We have verified that this length is sufficient to obtain the converged results for dispersion interaction for the relevant range of $\mathrm{H}_{2}$-CNT distances.

To use the analytic integration in $\theta$ and $\phi$ for dispersive part as well, as described in detail in [16], we first fit $V_{\mathrm{disp}-\mathrm{H}}(\rho)=\frac{1}{2} V_{\text {disp }-\mathrm{H}_{2}^{\prime \prime}}(\rho)$ as a polynomial function of $1 / R$,

$$
V_{\text {disp }-\mathrm{H}}(\rho)=\frac{p_{n}(1 / R)}{R^{5}}
$$

with $p_{n}$ being polynomials of order $n$. Explicit values of the $p_{n}$ coefficients are also provided in Appendix A, Tables A3, A4.

For arbitrary orientation of $\mathrm{H}_{2}$ molecule, using this fit and explicit expression of $\rho_{1,2}$ in terms of $R, \theta$ and $\phi$, the dispersive interaction was represented as

$$
V_{\mathrm{disp}}(R, x, \phi)=\sum_{i=0}^{m} \sum_{k=0}^{i} V_{\mathrm{disp}}^{i k}(R)\left(1-x^{2}\right)^{i} \cos ^{2 k}(\phi)
$$

We have found that the value $m=2$ is large enough for achieving an acceptable accuracy. Finally, each $R$-dependent factor $V_{\text {disp }}^{i k}(R)$ was fit to a polynomial form similar to that expressed in Eq. (7).

Using the Discrete Variable Representation (DVR) approach [47], the eigenvalues and eigenfunctions of the Hamiltonian were calculated as in our previous work [16], by direct diagonalization over a discrete basis built from sinc-DVR functions for $R$ and spherical harmonics for $\theta$ and $\phi, \chi_{i}(R) Y_{j m}(\theta, \phi)$.

From the form of the Hamiltonian, see Eq. (2), and the symmetry of the interaction reflected, e.g., in Eq. (8), it can be shown that the solutions have a few extra symmetries. For example, odd and even values of $j$ do not mix, as well as odd and even values of $m$. Moreover, for $\Lambda=0$, we have an extra symmetry condition with respect to the change of sign of $m$. For $\Lambda>0$ this is only approximate, but still remains good for the case of the $\mathrm{H}_{2}$ molecule rotating outside of the nanotube. For further details, we refer the interested reader to our previous work [16].

The parameters of the potential analitic representation, as mentioned above, are provided in the Appendix A, Tables A2-A4. For the dispersive contribution, we used a polynomial expansion of order 5 and 4 for the nanotubes of helicity indexes $(10,10)$ and $(5,5)$, respectively. In order to solve the eigenvalue problem, a radial DVR basis of sinc-DVR type was employed, with 91 basis function on the interval $[17,30]$ bohr for the tube $\operatorname{CNT}(10,10)$, and 85 basis functions on the interval $[9,30]$ bohr for the tube $\mathrm{CNT}(5,5)$. For both tubes, the angular basis was constructed from spherical harmonics with $j_{\max }=30$ and $m_{\max }=10$.

\subsection{Effective one-dimensional model}

Due to the large value of the rotational constant of the $\mathrm{H}_{2}$ molecule $\left(B_{0} \approx 59.3383\right.$ $\mathrm{cm}^{-1}$ ), the energy spacings between states with different $j$ values (i.e., $2 B_{0}, 6 B_{0}, \ldots$ ) are much larger than those associated to different $n$ values. This feature along with the symmetry of the problem suggests a very simple one-dimensional (1D) model to describe the $3 \mathrm{D}$ rotational wave functions. Assuming that $j$ is a good quantum number, the effective 1D potentials are built by averaging the full 3D Hamiltonian over the corresponding spherical harmonics. For $j=0$, we will have thus a single 1D potential to calculate. For $j=1$ (we consider here $\Lambda=0$ only, see below), all three components 
Table 1. SAPT(DFT)-based decomposition of the interaction energy between $\mathrm{H}_{2}$ and short nanotubes sCNTs of helicity indexes $(5,5)$ and $(10,10)$ at the potential minima position $(R=6.75$ an $10.5 \AA$, respectively). For comparison purposes, the energy values corresponding to $\mathrm{H}_{2}$ adsorption inside (the center) of the sCNT(5,5) are also included in parentheses, obtained following the procedure reported in Ref. 16. The angles $\theta$ and $\phi$ characterize the angular orientation of the $\mathrm{H}-\mathrm{H}$ internuclear axis with respect to the nanotube direction, as shown in Figure 1 (see also Figure 2).

\begin{tabular}{|c|c|c|c|c|c|c|}
\hline \multirow[b]{2}{*}{$(\theta, \phi)$} & \multicolumn{3}{|c|}{$(5,5)$} & \multicolumn{3}{|c|}{$(10,10)$} \\
\hline & $\left(0^{\circ}, 0^{\circ}\right)$ & $\left(90^{\circ}, 0^{\circ}\right)$ & $\left(90^{\circ}, 90^{\circ}\right)$ & $\left(0^{\circ}, 0^{\circ}\right)$ & $\left(90^{\circ}, 0^{\circ}\right)$ & $\left(90^{\circ}, 90^{\circ}\right)$ \\
\hline $\mathrm{E}_{\text {elec }}, \mathrm{meV}$ & $\begin{array}{c}-5.67 \\
(-57.77)\end{array}$ & $\begin{array}{c}-17.04 \\
(-51.42)\end{array}$ & -8.12 & -2.21 & -5.42 & -2.21 \\
\hline $\mathrm{E}_{\text {exch-rep }}, \mathrm{meV}$ & $\begin{array}{c}35.62 \\
(150.45)\end{array}$ & $\begin{array}{c}45.29 \\
(-179.08)\end{array}$ & 34.87 & 11.64 & 14.93 & 11.64 \\
\hline $\mathrm{E}_{\text {ind }}, \mathrm{meV}$ & $\begin{array}{l}-0.39 \\
(-2.80)\end{array}$ & $\begin{array}{l}-1.57 \\
(-5.05)\end{array}$ & -0.38 & -0.11 & -0.52 & -0.11 \\
\hline $\mathrm{E}_{\text {disp }}, \mathrm{meV}$ & $\begin{array}{c}-45.63 \\
(-235.80)\end{array}$ & $\begin{array}{c}-52.99 \\
(-254.10)\end{array}$ & -44.75 & -19.50 & -23.89 & -19.50 \\
\hline $\mathrm{E}_{\mathrm{tot}}, \mathrm{meV}$ & $\begin{array}{c}-16.07 \\
(-145.92)\end{array}$ & $\begin{array}{c}-26.31 \\
(-131.50)\end{array}$ & -18.38 & -10.18 & -14.90 & -10.17 \\
\hline
\end{tabular}

(i.e., $m=0, \pm 1$ ) do not interact. However, as explained in the Supplementary Material, the effective potentials are different, resulting in the splitting of the three components. To get the total energy for $j=1$, the $2 B_{0}$ contribution is simply added to the $1 \mathrm{D}$ energies of the $\mathrm{H}_{2}$ molecule moving as a whole in the effective $1 \mathrm{D}$ radial potential.

For $j>1$, some extra mixing between different $m$ states exists so that an explicit matrix form of the 1D Hamiltonian needs to be constructed and diagonalized. For example, $m=0$ and $m=+2$ do $\operatorname{mix}$ for $j=2$ (i.e., 1 and $\cos (2 \phi)$ functions). More details, as well as the discussion of the infinite CNT diameter limit (graphene) are provided in Supplementary Material.

\section{Results and discussion}

\subsection{Influence of the curvature of the nanotube on the angular dependence of the $\mathrm{H}_{2}$-nanotube interaction}

For short nanotubes of helicity indexes $(5,5)$ and $(10,10)$ and diameters of 6.7 and 13.6 $\AA$, Figure 2 shows the total $\mathrm{H}_{2} / \mathrm{sCNT}$ interaction potentials, for three orientations of the $\mathrm{H}_{2}$ molecule, as a function of the radial distance $R$ between the molecule centerof-mass and the nanotube center, with $\phi=0^{\circ}$ (see Figure 1). The electrostatic $\mathrm{E}_{\text {elec}}$, exchange-repulsion $\mathrm{E}_{\text {exch-rep }}$, induction $\mathrm{E}_{\text {ind }}$, and dispersion contributions $\mathrm{E}_{\text {disp }}$ are also presented, with the explicit energy values at the potential minima provided in Table 1. The configuration $\left(\theta=0^{\circ}, \phi=\right.$ any $)$, shown with full lines, correspond to a parallel orientation of the $\mathrm{H}_{2}$ to the tube long axis while the $\left(\theta=90^{\circ}, \phi=0^{\circ}\right)$ arrangement (dashed lines) is associated to a perpendicular configuration to tube long axis but along the radial direction. Finally, the $\mathrm{H}_{2}$ molecular is perpendicular to both the tube long axis and the radial direction when adopting the $\left(\theta=90^{\circ}, \phi=90^{\circ}\right)$ configuration (dotted lines).

As can be observed in Figure 2 and Table 1, the $\mathrm{H}_{2} / \mathrm{CNT}$ attractive interaction is dispersion-dominated while the net repulsive dispersionless contribution is mainly characterized by the exchange-repulsion term. This repulsion contribution grows exponentially as the distance between the $\mathrm{H}_{2}$ and the carbon cage decreases although 


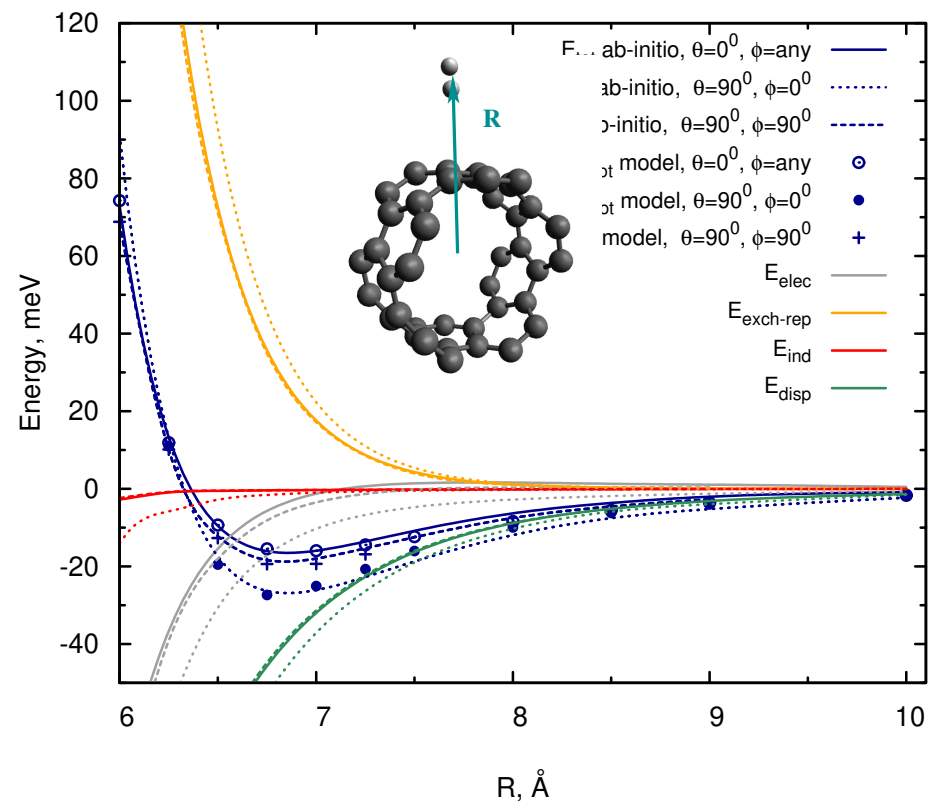

(a) $\mathrm{H}_{2} / \mathrm{sCNT}(5,5)$

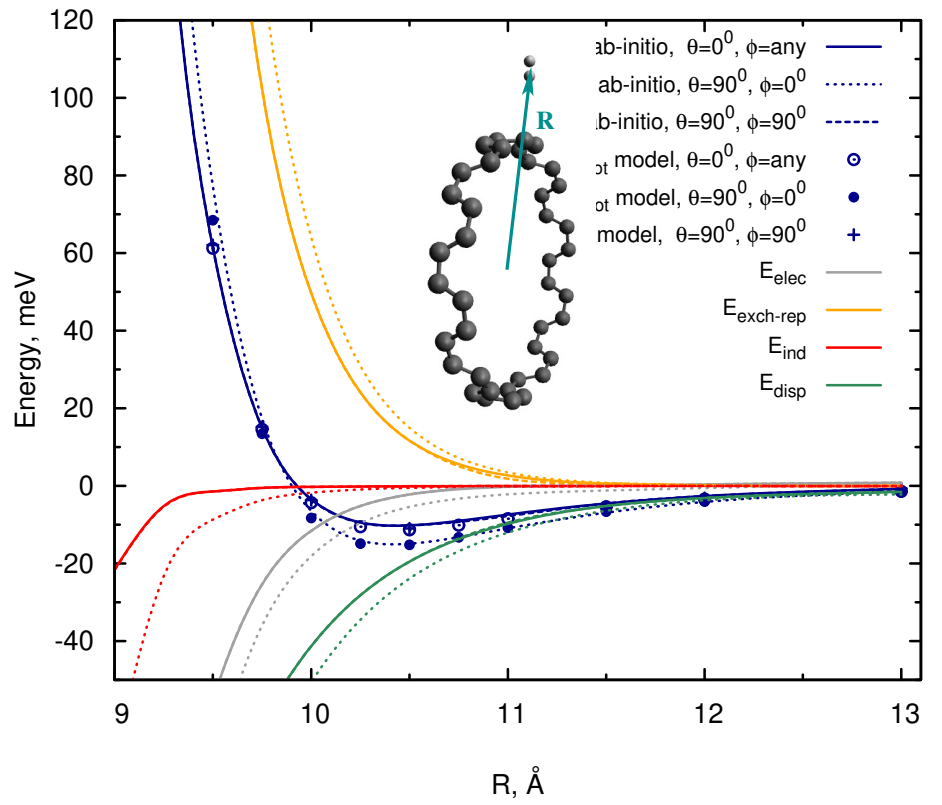

(b) $\mathrm{H}_{2} / \mathrm{sCNT}(10,10)$.

Figure 2. Radial scan of the total interaction energies between a single $\mathrm{H}_{2}$ molecule and short carbon nanotubes $(\mathrm{sCNT})$ of helicity indexes $(5,5)$, represented in the upper panel, and $(10,10)$, represented in the bottom panel. Gray spheres represent carbon atoms while white spheres stand for one $\mathrm{H}_{2}$ molecule. The interaction energies are represented as a function of the distance from the $\mathrm{H}_{2}$ center-of-mass to the nanotube axis, $R$. Full, dotted, and dashed lines correspond to $(\theta, \phi)$ values of $\left(0^{\circ}, 0^{\circ}\right),\left(90^{\circ}, 0^{\circ}\right)$ and $\left(90^{\circ}, 90^{\circ}\right)$, respectively, with the angles defined in Figure 1. The different SAPT-based energy contributions to the total interaction energy are also shown along with that obtained with our potential model. The $\mathrm{H}-\mathrm{H}$ bond length was fixed to the vibrationally averaged rigid rotor value, $\mathrm{r}_{0}=0.7508 \AA$ from Ref. 19. 
such behavior is smoothed out by the attractive electrostatic interaction, with the induction term contributing very little. All these features were also found for $\mathrm{H}_{2}$ located inside the CNTs (see Refs. 4, 16). However, in stark contrast with the latter, the perpendicular orientation to the tube axis but along the radial direction $\left(\theta=90^{\circ}\right.$, $\left.\phi=0^{\circ}\right)$, shown with dashed lines, is clearly energetically preferred over the parallel configuration $\left(\theta=0^{\circ}\right.$, full lines). This key difference arises from the major role of the electrostatic contribution: as opposed to the inside case, the electrostatic term is clearly more attractive when the $\mathrm{H}-\mathrm{H}$ axis is along the radial direction, compensating for the smaller exchange-repulsion when the molecule is located along the tube long axis. Since the dispersion contribution is also more attractive for the $\left(\theta=90^{\circ}, \phi=0^{\circ}\right)$ configuration, this arrangement is also energetically favored.

To further understand the very pronounced anisotropy of the electrostatic term when molecular hydrogen is located in the outside of CNTs, it is important to consider the existence of a curvature-induced dipole in the radial direction of the nanotube and oriented towards the outside of the carbon cage [34]. The electrostatic contribution becomes thus more attractive when the $\mathrm{H}-\mathrm{H}$ axis (i.e., the induced dipole) is oriented in the same direction of the curvature-dependent dipole of the nanotube as the polarizability of the hydrogen molecule along the molecular axis is about 1.5 times larger than the orthogonal one[48]. As the $\mathrm{H}_{2}$-nanotube distance decreases, the exchange-repulsion contribution grows much more rapidly than the electrostatic term. As a result (see Figure 2), the preferential orientation changes from perpendicular to parallel when entering into the repulsive region of the potential, where the exchangerepulsion contribution dominates.

Let us now discuss the dependence of the potential on the angle $\phi$ (as defined in Figure 1). For the tube $(5,5)$, it can be observed from Figure 2 and Table 1 that the net energy and individual energy contributions change very little in going from $\theta=0^{\circ}$ to the $\left(\theta=90^{\circ}, \phi=90^{\circ}\right)$ configuration. Once again, the most noticeable difference is for the electrostatic contribution, that is slightly more attractive when the molecule is not aligned with the tube long axis. For the wider tube $(10,10)$, the energy differences between $\theta=0^{\circ}$ and $\left(\theta=90^{\circ}, \phi=90^{\circ}\right)$ configurations become almost negligible. This is signaling that the value of the nanotube dipole decreases as its curvature becomes reduced upon increasing the diameter of the nanotube. Another consequence is that the anisotropy of the dispersionless $\mathrm{H}_{2}$-CNT interaction becomes determined mainly by the dispersion rather than the dispersionless contribution in going from $\mathrm{CNT}(5,5)$ to $\operatorname{CNT}(10,10)$ tubes.

\section{2. $\quad H_{2}$-nanotube potential model}

To consider longer nanotubes, we have applied the potential model described in section 2.2. It can be observed from Figure 2 that our model provides interaction energies agreeing very well with the SAPT(DFT)-based counterparts. By applying it to long nanotubes of helicity indexes $(5,5)$ and $(10,10)$, we obtain the dispersionless and dispersion interaction energies shown in Figure 3. Notice that the energy ordering of parallel and perpendicular configurations is not modified but the potential minima becomes significantly deeper due the the much more attractive dispersion contributions.

Our model has also been applied to describe the $\phi$-dependence of the interaction. For this purpose, we have chosen the orientation associated to the angles $(\theta, \phi)=\left(90^{\circ}, 90^{\circ}\right)$. This configuration corresponds to the $\mathrm{H}_{2}$ molecule lying perpendicular to both the CNT long axis and the CNT radius. As can be seen in Figure 3, its energy position is 


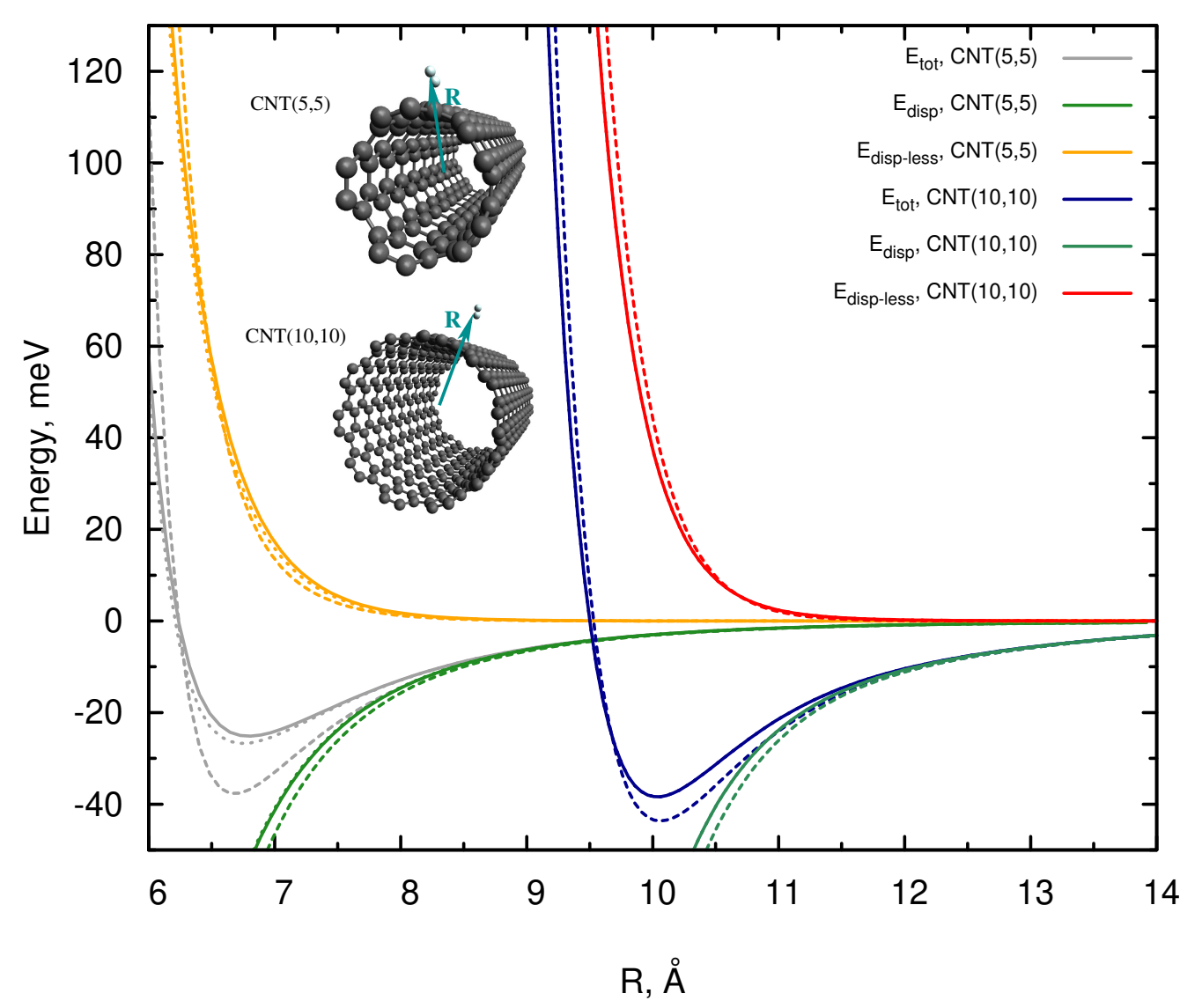

Figure 3. Radial scan of the total interaction energies between a single $\mathrm{H}_{2}$ molecule and long carbon nanotubes $(\mathrm{CNT})$ of helicity indexes $(5,5)$ and $(10,10)$, as calculated with our potential model. Gray spheres represent carbon atoms while white spheres stand for a single $\mathrm{H}_{2}$ molecule rotating outside the CNTs. Full, dashed, and dotted lines correspond to $(\theta, \phi)$ values of $\left(0^{\circ}, 0^{\circ}\right),\left(90^{\circ}, 0^{\circ}\right)$, and $\left(90^{\circ}, 90^{\circ}\right)$, respectively, with the angles defined in Figure 1. The dispersion and dispersionless energy contributions to the total interaction energies are also shown. $R$ is the distance from the $\mathrm{H}_{2}$ center-of-mass to the nanotube axis while $\theta$ is the angle between the $\mathrm{H}-\mathrm{H}$ internuclear axis and the tube long axis. 
in between those of parallel and perpendicular arrangements for the narrow $\mathrm{CNT}(5,5)$ tube but, for the wider $\operatorname{CNT}(10,10)$ tube, it is the highest-energy orientation. This change is a direct consequence of the less relevant role of the electrostatic contribution from the curvature-dependent nanotube dipole upon increasing its diameter.

\subsection{Molecular energy levels of $\mathrm{H}_{2}$ rotating in the outside of carbon nanotubes}

As illustrative examples, we have calculated the molecular energy levels of $\mathrm{H}_{2}$ located outside the nanotubes with helicity indexes $(5,5)$ and $(10,10)$. These energy levels are assigned using three quantum numbers, $n$ for the radial $R$ degree of freedom, $j$ for the $\mathrm{H}_{2}$ rotation associated with $x=\cos (\theta)$, and $m$ for the angular momentum projection quantum number corresponding to $\phi$. The energy levels and wavefunctions were calculated by diagonalizing the $3 \mathrm{D} \Lambda$-dependent Hamiltonian, see Eq. (1), as described above. The dependence on $\Lambda$ is very weak (perturbative) due to the large values of the equilibrium $R$ distances (see Figure 2), resulting in small values of the overall rotational constant (below $0.1 \mathrm{~cm}^{-1}$ ):

$$
B_{\mathrm{ext}}=\frac{1}{2 M}\left\langle\frac{1}{R^{2}}\right\rangle .
$$

As a consequence, the energy levels for $\Lambda>0$ are perfectly fitted through the expression,

$$
E(\Lambda)=E(\Lambda=0)+B_{\text {ext }} \Lambda^{2}
$$

The energy levels $E(\Lambda=0)$ as well as the values of $B_{\text {ext }}$ are presented in Table 2 for $n<4$, and $j, m<3$. For comparison purposes, the energy values obtained without accounting for the rotation are also provided. These energy levels were obtained by diagonalizing the $1 \mathrm{D}$ radial Hamiltonian describing the motion of $\mathrm{H}_{2}$ as a whole (as in Ref. 4), employing the potential associated to the parallel orientation, $V(R, \theta=0)$.

As mentioned in section 2.3, the whole Hamiltonian has extra symmetries. First, the states with odd and even values of $j$ do not mix, corresponding to the symmetry transformation $\theta \rightarrow \pi-\theta$ (or $x \rightarrow-x$ ). Second, odd and even values of $m$ do not mix either, reflecting that the Hamiltonian is invariant under the symmetry operation $\phi \rightarrow \phi+\pi$. Finally, for $\Lambda=0$, symmetric and antisymmetric combinations of $m$ and $-m$ do not mix due to the Hamiltonian invariance under the symmetry transformation $\phi \rightarrow-\phi$. In Table 2, $+m$ and $-m$ corresponds to symmetric and antisymmetric combinations, with the $\phi$-dependence described as $\cos (m \phi)$ and $\sin (m \phi)$, respectively. This symmetry condition is not exact for $\Lambda>0$ but still quite accurate.

It can be observed from Table 2 that zero-point energies are rather large and that the vibrational $R$ motion is quite anharmonic, as expected for a light molecule such as $\mathrm{H}_{2}$. As for the inside location [4], the energy differences with and without including the rotation for the $\mathrm{H}_{2}$ center-of-mass motion are very pronounced for the molecule in the outside of the tube $(5,5)$ with diameter below one nanometer and are smaller for the tube $(10,0)$ with diameter above one nanometer.

The results obtained using effective 1D model, are also provided in Table 2. By analyzing the contribution of different angular components of the potential, see Eq. (6), we found that the terms with $i \leq 1$ are the most important while higher-order contributions can be practically neglected. Energy levels for this simple 1D model were 
Table 2. Energy levels (in $\mathrm{cm}^{-1}$ ) of a rotating $\mathrm{H}_{2}$ molecule located outside of a nanotube of helicity indices $(5,5)$ and $(10,10)$, for $n \leq 3, j,|m| \leq 2$ obtained using full 3D calculation and $1 \mathrm{D}$ model. The energies at the potential minima $\left(E_{\mathrm{min}}\right.$, in $\left.\mathrm{cm}^{-1}\right)$ and the zero-point energies $\left(E_{\mathrm{zp}}\right.$, in $\left.\mathrm{cm}^{-1}\right)$ are also collected. The energy values are relative to that of the ground state $\left(\begin{array}{lll}0 & 0 & 0\end{array}\right)$. The energy of the ground state $\left(\begin{array}{lll}0 & 0 & 0\end{array}\right)$ is given between parenthesis. The dependence on $\Lambda$ is described with the external rotation constant $B_{\text {ext }}$, with their values being also listed. For comparison, we provide the energy values without including the rotational mode (in square brackets).

\begin{tabular}{|c|c|c|c|c|c|c|c|c|}
\hline & & & & $\mathrm{TT}(5,5)$ & & $\mathrm{Cl}$ & $\Gamma(10,10)$ & \\
\hline$d_{\mathrm{CNT}}, \AA$ & & & & 6.7 & & & 13.6 & \\
\hline$E_{\min }, \mathrm{cm}^{-1}$ & & & & 04.386 & & & 352.10 & \\
\hline$E_{\mathrm{zp}}, \mathrm{cm}^{-1}$ & & & & 3.491 & & & 02.75 & \\
\hline$n$ & $j$ & $m$ & $\mathrm{E}_{\Lambda=0}$ & $\mathrm{E}_{1 D}$ & $B_{\text {ext }}$ & $\mathrm{E}_{\Lambda=0}$ & $\mathrm{E}_{1 D}$ & $B_{\text {ext }}$ \\
\hline 0 & 0 & 0 & $\begin{array}{c}0.000 \\
(-188.395) \\
{[-159.307]}\end{array}$ & 0.000 & 0.176 & $\begin{array}{c}0.000 \\
(-260.435) \\
{[-249.881]}\end{array}$ & 0.000 & 0.081 \\
\hline 0 & 1 & 0 & 131.048 & 129.881 & 0.174 & 123.120 & 122.585 & 0.081 \\
\hline 0 & 1 & 1 & 100.429 & 99.949 & 0.172 & 108.685 & 108.940 & 0.079 \\
\hline 0 & 1 & -1 & 127.135 & 125.690 & 0.180 & 125.102 & 124.448 & 0.082 \\
\hline 0 & 2 & 0 & 370.830 & 369.121 & 0.179 & 363.794 & 362.982 & 0.084 \\
\hline 0 & 2 & 1 & 352.088 & 350.973 & 0.169 & 352.168 & 351.899 & 0.078 \\
\hline 0 & 2 & -1 & 370.717 & 369.015 & 0.181 & 363.732 & 362.941 & 0.083 \\
\hline 0 & 2 & 2 & 344.962 & 342.582 & 0.092 & 349.331 & 349.039 & 0.065 \\
\hline 0 & 2 & -2 & 349.623 & 347.831 & 0.253 & 353.815 & 353.221 & 0.093 \\
\hline 1 & 0 & 0 & $\begin{array}{c}79.640 \\
{[67.015]}\end{array}$ & 78.768 & 0.156 & $\begin{array}{c}98.629 \\
{[95.243]}\end{array}$ & 98.313 & 0.075 \\
\hline 1 & 1 & 0 & 205.259 & 203.814 & 0.154 & 220.317 & 219.673 & 0.075 \\
\hline 1 & 1 & 1 & 187.919 & 186.828 & 0.151 & 210.308 & 210.201 & 0.074 \\
\hline 1 & 1 & -1 & 202.993 & 201.327 & 0.162 & 221.749 & 221.001 & 0.077 \\
\hline 1 & 2 & 0 & 443.952 & 442.164 & 0.161 & 459.998 & 459.147 & 0.079 \\
\hline 1 & 2 & 1 & 433.422 & 431.975 & 0.147 & 451.925 & 451.457 & 0.073 \\
\hline 1 & 2 & -1 & 443.889 & 442.099 & 0.163 & 459.949 & 459.117 & 0.078 \\
\hline 1 & 2 & 2 & 429.116 & 427.184 & 0.057 & 450.173 & 449.474 & 0.055 \\
\hline 1 & 2 & -2 & 432.145 & 430.135 & 0.248 & 453.163 & 452.406 & 0.092 \\
\hline 2 & 0 & 0 & $\begin{array}{c}132.368 \\
{[112.472]}\end{array}$ & 131.091 & 0.134 & $\begin{array}{c}166.359 \\
{[160.041]}\end{array}$ & 165.830 & 0.069 \\
\hline 2 & 1 & 0 & 254.907 & 253.324 & 0.132 & 286.823 & 286.102 & 0.069 \\
\hline 2 & 1 & 1 & 245.278 & 243.869 & 0.127 & 280.754 & 280.353 & 0.066 \\
\hline 2 & 1 & -1 & 253.641 & 251.886 & 0.142 & 287.774 & 286.963 & 0.071 \\
\hline 2 & 2 & 0 & 493.009 & 491.199 & 0.141 & 525.642 & 524.766 & 0.074 \\
\hline 2 & 2 & 1 & 487.187 & 485.570 & 0.122 & 520.710 & 520.081 & 0.065 \\
\hline 2 & 2 & -1 & 492.981 & 491.160 & 0.145 & 525.603 & 524.745 & 0.072 \\
\hline 2 & 2 & 2 & 484.966 & 482.892 & 0.006 & 519.477 & 518.884 & 0.045 \\
\hline 2 & 2 & -2 & 486.599 & 484.520 & 0.254 & 521.578 & 520.702 & 0.087 \\
\hline 3 & 0 & 0 & $\begin{array}{c}164.642 \\
{[140.297]}\end{array}$ & 163.133 & 0.110 & $\begin{array}{c}209.099 \\
{[200.801]}\end{array}$ & 208.441 & 0.061 \\
\hline 3 & 1 & 0 & 285.321 & 283.654 & 0.108 & 328.735 & 327.964 & 0.061 \\
\hline 3 & 1 & 1 & 280.356 & 278.745 & 0.101 & 325.431 & 324.828 & 0.057 \\
\hline 3 & 1 & -1 & 284.674 & 282.879 & 0.119 & 329.337 & 328.494 & 0.064 \\
\hline 3 & 2 & 0 & 523.073 & 521.255 & 0.120 & 566.956 & 566.067 & 0.070 \\
\hline 3 & 2 & 1 & 520.094 & 518.372 & 0.094 & 564.209 & 563.472 & 0.056 \\
\hline 3 & 2 & -1 & 523.062 & 521.233 & 0.123 & 566.924 & 566.052 & 0.066 \\
\hline 3 & 2 & 2 & 519.006 & 516.968 & -0.037 & 563.604 & 562.829 & 0.029 \\
\hline 3 & 2 & -2 & 519.890 & 517.826 & 0.245 & 564.798 & 563.855 & 0.084 \\
\hline
\end{tabular}




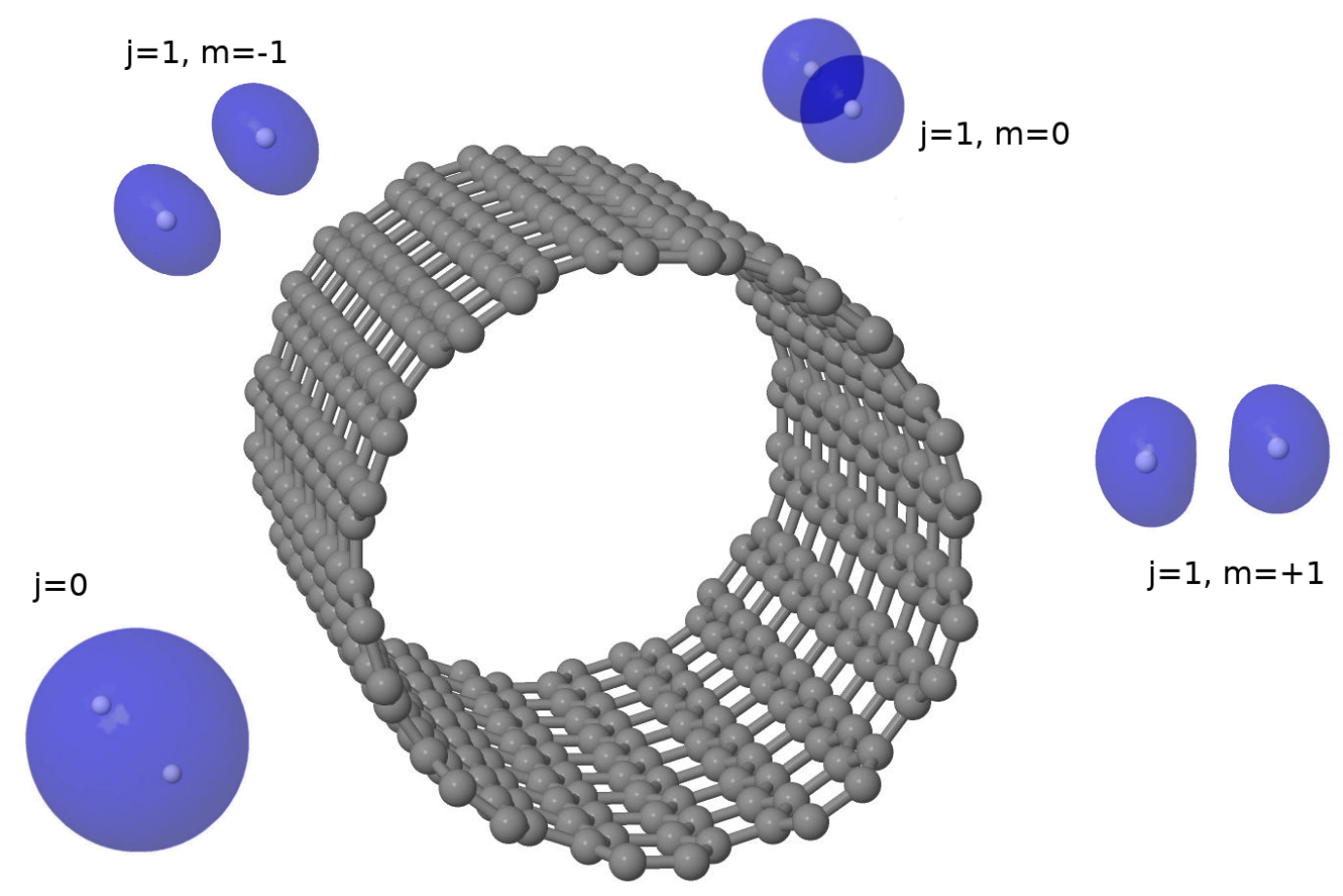

Figure 4. Figure illustrating the states associated to $j=0$, and the three components $(m=0, \pm 1)$ of the $j=1$ state.

obtained including only $i \leq 1$ terms in the potential. As can be seen, the model reproduce almost exactly (to within $1 \%$ ) the $3 \mathrm{D}$ values. The corresponding effective potentials are shown in the Supporting Material along with their correlation with the $\mathrm{H}_{2}$-nanotube interaction potentials in different orientations.

Actually, as illustrated in Figure 4, the three states associated to $j=1$ have a very clear physical interpretation: they simply represent three orthogonal symmetric orientation of the $\mathrm{H}_{2}$ molecule. Thus, the state with $m=0$ corresponds to a parallel orientation of the $\mathrm{H}_{2}$ molecule (i.e, along the $Z$ axis); the state with $m=+1$ describes the $\mathrm{H}_{2}$ orientation along the radial direction connecting the CNT center with the $\mathrm{H}_{2}$ center-of-mass (i.e., the $X$ axis for $\Phi=0$ ). Finally, for $m=-1, \mathrm{H}_{2}$ is lying perpendicular to both the CNT long axis and to the radial direction (i.e., the $Y$ axis).

The analysis of the splitting of $j=1$ levels shows very interesting physical effects. First, this splitting is very sensitive to the representation of the potential. In our preliminary study for the outside $\mathrm{H}_{2}$ location [16], we used a $\rho$-only dependent model for both dispersionless and dispersive contributions. This resulted in the degeneracy of $j=1, m=0$ and $j=1, m=-1$ levels, with the level $j=1, m=+1$ lying higher in energy (see Supplementary Material of Ref. 16). In the present work, we have found that this model is not accurate enough for the dispersionless contribution due to an enhanced role of the electrostatic term. More specifically, due to the interaction of $\mathrm{H}_{2}$ with the induced dipole of the nanotube, the radially aligned $j=1, m=+1$ state is the lowest. Moreover, the energy ordering of the states with $j=1, m=0$ and $j=1, m=-1$ is modified when going from the $\operatorname{CNT}(5,5)$ to the $\operatorname{CNT}(10,10)$ tube: for the $\operatorname{CNT}(5,5)$ tube $j=1, m=0$ is the highest in energy while, for the CNT $(10,10)$ tube it is lower than $j=1, m=-1$ However, in both cases the splitting 
between $j=1, m=0$ and $j=1, m=-1$ levels is much smaller than that between $j=1, m=0$ and $j=1, m=+1$ reflecting the fact that it should disapper for larger nanotube diameter as discussed in the next section.

\subsection{Comparison with the $\mathrm{H}_{2} /$ graphene system}

It is interesting to analyze our results by considering the limit of an infinite CNT radius which corresponds to interaction of the $\mathrm{H}_{2}$ molecule with the graphene surface, as analyzed in our previous work [19]. We notice, however, that the axes are oriented differently so that the $m$ quantum numbers have different meaning. For the interaction with graphene, the $Z$ axis was oriented perpendicularly to the surface, i.e. it corresponds to the $X$ axis in the present study (see Figure 1). Specifically, the $X$ and $Y$ axes for graphene translate into the $Y$ and $Z$ axes in the present case. As a consequence, the $m=0,+1,-1$ values in the graphene case [19] would correspond to the $m=+1,0,-1$ values in the present work. For graphene, the $m= \pm 1$ states are degenerate, reflecting the isotropy of the surface, while the $m=0$ state is the lowest-energy state. It correlates well with the present finding on the $m=1$ state as the lowest-energy one. However, the $m=0$ and $m=-1$ states are still not degenerate due to the curvature of the carbon cage.

Upon increasing the value of the CNT diameter, the graphene $m= \pm 1$ states should become progressively more and more degenerate. The values of the $m$-splittings in the present work are about $30 \mathrm{~cm}^{-1}$ for CNT $(5,5)$ and $15 \mathrm{~cm}^{-1}$ for CNT $(10,10)$ and are similar to those previously found for graphene and graphite (ca. $23 \mathrm{~cm}^{-1}$ from Ref. 19). Both values are somewhat larger than those measured for aligned carbon nanotubes $(8$ $\mathrm{cm}^{-1}$ from Ref. 14). The difference might be due to an additional confinement effect induced by the alignment of carbon nanotubes. Moreover, since the $m$-splitting values are very sensitive to both the $\theta$ - and $\phi$-dependence of the dispersionless interaction, work is in progress to ensure its correct treatment in wider nanotubes.

\section{Conclusions}

To summarize, this study has revealed the impact of the anisotropy of the $\mathrm{H}_{2}$-nanotube interaction on the molecular rotation in the outside of both sub- and nanometer-sized carbon nanotubes CNTs. In particular, in constrast with the case of the $\mathrm{H}_{2}$ location inside the CNTs [16], a SAPT(DFT)-based energy decomposition has shown a marked influence of the electrostatic contribution in the balance determining the anisotropy of the interaction in a narrow nanotube. This feature has been interpreted as an indication of the key role of the curvature-dependent dipole of the nanotube. This way, our results indicate that there is a strong correlation between the curvatureinduced dipole of carbon nanotubes, the anisotropy of the $\mathrm{H}_{2}$-nanotube interaction, and the energy levels landscape. Moreover, we propose an effective one-dimensional model for the nuclear motion capable of reproducing full-dimensional approach energy levels to within $1 \%$.

\section{Acknowledgements}

This work has been partly supported by the Spanish Agencia Estatal de Investigación (AEI) and the Fondo Europeo de Desarrollo Regional (FEDER, UE) under Grant 
No. MAT2016-75354-P and COST Action CM1405 "Molecules in Motion" (MOLIM). MPdLC is greatly thankful to the CTI (CSIC) and CESGA super-computer facilities (Spain) for the provided computational resources.

\section{References}

[1] H. M. Cheng, Q. H. Yang and C. Liu, Carbon, 2001, 39, 1447-1454.

[2] S. Kumar, R. Rani, N. Dilbaghi, K. Tankeshwar and K.-H. Kim, Chem. Soc. Rev., 2017, 46, 158-196.

[3] V. P. Ting, A. J. Ramirez-Cuesta, N. Bimbo, J. E. Sharpe, A. Noguera-Diaz, V. Presser, S. Rudic and T. J. Mays, ACS Nano, 2015, 9, 8249-8254.

[4] M. P. de Lara-Castells, A. W. Hauser, A. O. Mitrushchenkov and R. Fernández-Perea, Phys. Chem. Chem. Phys., 2017, 19, 28621-28629.

[5] A. Bazargan and G. McKay, Chemical Engineering Journal, 2012, 195-196, 377 - 391.

[6] D. Yao, Y. Zhang, P. T. Williams, H. Yang and H. Chen, Applied Catalysis B: Environmental, 2018, 221, $584-597$.

[7] A. W. Hauser and M. P. de Lara-Castells, J. Phys. Chem. Lett., 2016, 7, 4929-4935.

[8] A. W. Hauser, A. O. Mitrushchenkov and M. P. de Lara-Castells, J. Phys. Chem. C, 2017, 121, 3807-3821.

[9] M. Rossi and F. Ancilotto, Phys. Rev. B, 2016, 94, 100502.

[10] G. Ferré, M. C. Gordillo and J. Boronat, Phys. Rev. B, 2017, 95, 064502.

[11] A. Del Maestro and M. Boninsegni, Phys. Rev. B, 2017, 95, 054517.

[12] T. X. Nguyen, H. Jobic and S. K. Bhatia, Phys. Rev. Lett., 2010, 105, 085901.

[13] M. Mondelo-Martell and F. Huarte-Larrañaga, J. Phys. Chem. A, 2016, 120, 6501-6512.

[14] H. G. Schimmel, G. J. Kearley and F. M. Mulder, Chem. Phys. Chem. Comm., 2004, 5, $1053-1055$.

[15] F. Fernández-Alonso, F. J. Bermejo, C. Cabrillo, R. O. Loutfy, V. Leon and M. L. Saboungi, Phys. Rev. Lett., 2007, 98, 215503.

[16] M. P. de Lara-Castells and A. O. Mitrushchenkov, Phys. Chem. Chem. Phys., 2018.

[17] D. G. A. Smith and K. Patkowski, J. Phys. Chem. C, 2014, 118, 544-550.

[18] D. G. A. Smith and K. Patkowski, J. Phys. Chem. C, 2015, 119, 4934-4948.

[19] M. P. de Lara-Castells and A. O. Mitrushchenkov, J. Phys. Chem. A, 2015, 119, 11022 11032.

[20] M. d. R. Cuevas-Flores, M. A. García Revilla and M. Bartolomei, J. Comp. Chem, 2018, 39, $71-80$.

[21] M. Bartolomei, R. Perez de Tudela, K. Arteaga, T. González Lezana, M. I. Hernandez, J. Campos-Martínez, P. Villarreal, J. Hernández Rojas, J. Breton and F. Pirani, Phys. Chem. Chem. Phys., 2017, 19, 26358-26368.

[22] M. Bartolomei, E. Carmona-Novillo, M. I. Hernández, J. Campos-Martínez and F. Pirani, J. Phys. Chem. C, 2013, 117, 10512-10522.

[23] A. Hesselmann and T. Korona, Phys. Chem. Chem. Phys., 2011, 13, 732-743.

[24] A. W. Hauser and M. P. de Lara-Castells, Phys. Chem. Chem. Phys., 2017, 19, 1342-1351.

[25] H. Stoll, J. Chem. Phys., 1992, 97, 8449-8454.

[26] E. Voloshina, D. Usvyat, M. Schütz, Y. Dedkov and B. Paulus, Phys. Chem. Chem. Phys., 2011, 13, 12041-12047.

[27] M. P. de Lara-Castells, H. Stoll, B. Civalleri, M. Causà, E. Voloshina, A. O. Mitrushchenkov and M. Pi, J. Chem. Phys., 2014, 141, 151102.

[28] M. P. de Lara-Castells, A. O. Mitrushchenkov and H. Stoll, J. Chem. Phys., 2015, 143, 102804.

[29] M. P. de Lara-Castells, N. F. Aguirre, H. Stoll, A. O. Mitrushchenkov, D. Mateo and M. Pi, J. Chem. Phys., 2015, 142, 131101.

[30] M. P. de Lara-Castells, M. Bartolomei, A. O. Mitrushchenkov and H. Stoll, J. Chem. 
Phys., 2015, 143, 194701.

[31] K. Pernal, R. Podeszwa, K. Patkowski and K. Szalewicz, Phys. Rev. Lett., 2009, 103, 263201.

[32] R. Podeszwa and K. Szalewicz, J. Chem. Phys., 2012, 136, 161102.

[33] R. Podeszwa, K. Pernal, K. Patkowski and K. Szalewicz, J. Phys. Chem. Lett., 2010, 1, $550-555$.

[34] T. Dumitrică, C. M. Landis and B. I. Yakobson, Chem. Phys. Lett., 2002, 360, 182-188.

[35] Z. Qiuchen and Z. Jin, Small, 10, 4586-4605.

[36] A. J. Misquitta, B. Jeziorski and K. Szalewicz, Phys. Rev. Lett., 2003, 91, 033201.

[37] A. Heßelmann and G. Jansen, Chem. Phys. Lett., 2003, 367, 778-784.

[38] H.-J. Werner, P. J. Knowles, G. Knizia, F. R. Manby, M. Schütz and others, MOLPRO, version 2012.1, a package of ab initio programs, see http://www.molpro.net.

[39] A. Heßelmann, G. Jansen and M. Schütz, J. Chem. Phys., 2005, 122, 014103.

[40] J. P. Perdew, K. Burke and M. Ernzerhof, Phys. Rev. Lett., 1996, 77, 3865-3868.

[41] D. E. Woon and T. H. Dunning, Jr., J. Chem. Phys., 1994, 100, 2975-2988.

[42] F. Weigend, Phys. Chem. Chem. Phys., 2002, 4, 4285-4291.

[43] F. Weigend, A. Köhn and C. Hättig, J. Chem. Phys., 2002, 116, 3175-3183.

[44] M. Grüning, O. V. Gritsenko, S. V. A. van Gisbergen and E. J. Baerends, J. Chem. Phys., 2001, 114, 652-660.

[45] S. Lias, in NIST Chemistry WebBook, NIST Standard Reference Database Number 69, ed. P. Linstrom and W. Mallard, National Institute of Standards and Technology, Gaithersburg, 2005, ch. Ionization Energy Evaluation.

[46] C. Adamo and V. Barone, J. Chem. Phys., 1999, 110, 6158-6170.

[47] Z. Bačic̀ and J. C. Light, Annu. Rev. Phys. Chem., 1989, 40, 469-498.

[48] W. Kołos and L. Wolniewicz, The Journal of Chemical Physics, 1967, 46, 1426-1432.

[49] K. T. Tang and J. P. Toennies, J. Chem. Phys., 1984, 80, 3726-3741.

\section{Appendix A. Parameters for the $\mathbf{H}_{2}$-nanotube dispersion and dispersionless interaction energies}

The additive pairwise potential model (PPM) proposed in Ref. 4 has been used to fit the dispersion contribution to the $\mathrm{H}_{2}$-nanotube interaction energy $E_{\text {int }}^{\text {disp }}$. Specifically, the dispersion energies calculated with the SAPT(DFT) method were fitted by means of the $\mathrm{D}_{a s}$ functional of Szalewicz and collaborators [31-33] but modulated by a corrugation scaling amplitude:

$$
\begin{array}{r}
E_{\mathrm{int}}^{\mathrm{disp}}\left(\left\{\mathbf{R}_{\mathrm{A}-\mathrm{C}}\right\}\right)=-\sum_{\mathrm{C}}\left[1+\gamma_{A}\left(1-\frac{3}{2} \cos ^{2} \theta_{\mathrm{C}}\right)\right] \\
\times \sum_{n=6,8} \frac{\sqrt{C_{n}^{\mathrm{A}} C_{n}^{\mathrm{C}}}}{R_{\mathrm{A}-\mathrm{C}}^{n}} f_{n}\left(\sqrt{\beta_{\mathrm{A}} \beta_{\mathrm{C}}} R_{\mathrm{A}-\mathrm{C}}\right),
\end{array}
$$

where $R_{\mathrm{A}-\mathrm{C}}$ stands for the distance between the $\mathrm{H}_{2}$ center-of-mass and one carbon atom, $\theta_{\mathrm{C}}$ is the angle between the vector going from the nanotube center to the carbon atom and the vector $\mathbf{R}_{\mathrm{A}-\mathrm{C}}$ pointing from the adsorbate center-of-mass to the same carbon atom. The sum in the second term (the $\mathrm{D}_{a s}$ function) runs over all carbon atoms of the nanotube and the terms $f_{n}$ stand for the damping functions of Tang and Toennies [49]. The dimensionless factor $\gamma_{A}$ in the first term measures the anticorrugation strength, when $\gamma_{A}$ bears a negative value, indicating that the dispersion become more attractive when the $\mathrm{H}_{2}$ molecule is on top of one carbon atom of the 
Table A1. Parameters defining the dispersion contributions to the $\mathrm{H}_{2} / \mathrm{CNT}$ interaction energies using the additive pairwise potential model (PPM) proposed in Ref. 4.

\begin{tabular}{lcccc}
\hline & \multicolumn{4}{c}{ Dispersion parameters } \\
& $C_{6}^{X} / \mathrm{meV} \cdot \AA^{6}$ & $C_{8}^{X} / \mathrm{meV} \cdot \AA^{8}$ & $\beta^{X} / \AA^{-1}$ & $\gamma_{A}$ \\
\cline { 2 - 5 } $\mathrm{X}=\mathrm{C}$ & 12985.1 & 0.0 & 4.7307 & -0.4284 \\
$\mathrm{X}=\mathrm{H}_{2}$ & 23940.6 & 199945 & 4.3263 & - \\
\hline
\end{tabular}

nanotube. The parameters of the PPM model are provided in Table A1. It can be noted that only the values of the $C_{6}^{\mathrm{H}_{2}}$ and $\beta^{\mathrm{H}_{2}}$ parameters differ from to those given in Ref. 4 and calculated with the $\mathrm{H}_{2}$ molecule located inside the $\mathrm{CNT}(5,5)$ tube.

Table A2 shows the parameters characterizing the dispersionless $\mathrm{H}_{2}$-nanotube interaction (see section 2.2), while Tables A3 and A4 provide parameters of dispersive part. We note that for $\operatorname{CNT}(10,10)$ the dispersionless potentials for $\theta=0^{\circ}$ and $\theta=90^{\circ}, \phi=90^{\circ}$ are extremely close so we have taken the same parameters for the fit, see Table A2. Thus, the term $V^{10}$ vanishes in this case. For CNT $(5,5)$ there difference is larger and so parameters differ.

We recall that parameters of dispersion interaction given in Table A1, refer to PPM model for individual $\mathrm{C}-\mathrm{H}$ interaction. The orientation dependent $\mathrm{H}_{2}$-(infinite)CNT dispersion interaction is obtained by summation over all CNT atoms (taken as long as needed to ensure convergence) and the subsequent fitting. The results of this fitting are presented in Tables A3, A4 and the dispersion part is obtained explicitly from these coefficients as

$$
V_{\text {disp }}(R, x, \phi)=\sum_{i=0}^{m} \sum_{k=0}^{i} V_{\text {disp }}^{i k}(R)\left(1-x^{2}\right)^{i} \cos ^{2 k}(\phi)
$$

where

$$
V_{\mathrm{disp}}^{i k}(R)=\frac{\sum_{j=0}^{n} t_{j}^{i k}\left(\frac{1}{R}\right)^{j}}{R^{5}}
$$

The dispersionless part is obtained by a sum similar to Eq.(A2) with $m=1$, where $V_{\text {disp-less }}^{i k}(R)$ are obtained as linear combinations of corresponding potentials at three different orientations, according to Eq.(5), that, in turn, have explicit analytic expression Eq.(6) with parameters given in Table A2. 
Table A2. Parameters defining the dispersionless contributions to the $\mathrm{H}_{2} / \mathrm{CNT}$ interaction energies for the nanotubes of helicity indexes $(5,5)$ and $(10,10)$.

\begin{tabular}{lccc}
\hline & \multicolumn{1}{c}{$\mathrm{CNT}(5,5)$} & & \\
$\theta=0^{\circ}$ & $\mathrm{A} / \mathrm{meV}$ & 2.2772 & $\beta / \AA^{-2}$ \\
\cline { 2 - 4 }$\theta=90^{\circ}, \phi=0^{\circ}$ & $1.42016 \cdot 10^{8}$ & 2.3251 & 0.4117 \\
$\theta=90^{\circ}, \phi=90^{\circ}$ & $1.42014 \cdot 10^{8}$ & 2.2902 & 0.3770 \\
\hline & $1.42014 \cdot 10^{8}$ & & 0.4092 \\
\hline & $\mathrm{CNT}(10,10)$ & $\alpha / \AA^{-1}$ & $\beta / \AA^{-2}$ \\
$\theta=0^{\circ}$ & $\mathrm{A} / \mathrm{meV}$ & 1.6959 & 0.1532 \\
$\theta=90^{\circ}, \phi=0^{\circ}$ & $1.42009 \cdot 10^{8}$ & 1.8577 & 0.1501 \\
$\theta=90^{\circ}, \phi=90^{\circ}$ & $1.42009 \cdot 10^{8}$ & 1.6959 & 0.1532 \\
\hline
\end{tabular}

Table A3. Parameters of the fits to account for the angular dependent interaction, Eq. (6), such as $V_{d i s p}^{i k}(R)=$ $p_{n}^{i k}(1 / R) / R^{5}$ with $p_{n}^{i k}(x)=\sum_{j=0}^{n} t_{j}^{i k} x^{j}$, for the nanotube CNT(5,5). Energy values are given in meV and distances are provided in $\AA$. Powers of 10 are given in parentheses. We note that the $p_{n}$ coefficients appearing in Eq. (7) (parallel orientation) are exactly the same as for the $p^{00}$ case below.

\begin{tabular}{ccccccc}
\hline$j$ & 0 & 1 & 2 & 3 & 4 & 5 \\
\hline \hline$t_{j}^{00}$ & $-0.5560965(7)$ & $0.2443774(9)$ & $-0.4226421(10)$ & $0.3494356(11)$ & $-0.1393687(12)$ & $0.2080465(12)$ \\
$t_{j}^{10}$ & $0.2130418(6)$ & $-0.9412775(7)$ & $0.1600929(9)$ & $-0.1309009(10)$ & $0.5127714(10)$ & $-0.7507330(10)$ \\
$t_{j}^{11}$ & $-0.3025122(7)$ & $0.1358180(9)$ & $-0.2360527(10)$ & $0.1986538(11)$ & $-0.8078217(11)$ & $0.1249451(12)$ \\
$t_{j}^{20}$ & $-0.5657917(4)$ & $0.2431584(6)$ & $-0.4021383(7)$ & $0.3201542(8)$ & $-0.1222434(9)$ & $0.1764795(9)$ \\
$t_{j}^{21}$ & $0.2875362(6)$ & $-0.1249993(8)$ & $0.2100560(9)$ & $-0.1710924(10)$ & $0.6758078(10)$ & $-0.1031020(11)$ \\
$t_{j}^{22}$ & $-0.8673363(6)$ & $0.3794565(8)$ & $-0.6428611(9)$ & $0.5291679(10)$ & $-0.2119686(11)$ & $0.3297781(11)$ \\
\hline
\end{tabular}

Table A4. Parameters of the fits to account for the angular dependent interaction, Eq. (6), such as $V_{d i s p}^{i k}(R)=$ $p_{n}^{i k}(1 / R) / R^{5}$ with $p_{n}^{i k}(x)=\sum_{j=0}^{n} t_{j}^{i k} x^{j}$, for the nanotube $\mathrm{CNT}(10,10)$. Energy values are given in meV and distances are provided in $\AA$. Powers of 10 are given in parentheses. We note that the $p_{n}$ coefficients appearing in Eq. (7) (parallel orientation) are exactly the same as for the $p^{00}$ case below.

\begin{tabular}{cccccc}
\hline$j$ & 0 & 1 & 2 & 3 & 4 \\
\hline \hline$t_{j}^{00}$ & $-0.1260955(9)$ & $0.7764788(10)$ & $-0.1738772(12)$ & $0.1686217(13)$ & $-0.6054142(13)$ \\
$t_{j}^{10}$ & $0.4757973(7)$ & $-0.2449426(9)$ & $0.4745701(10)$ & $-0.4103420(11)$ & $0.1338520(12)$ \\
$t_{j}^{11}$ & $-0.5791307(8)$ & $0.3008662(10)$ & $-0.5906302(11)$ & $0.5198074(12)$ & $-0.1733430(13)$ \\
$t_{j}^{20}$ & $-0.5691953(5)$ & $0.2835595(7)$ & $-0.5303355(8)$ & $0.4418789(9)$ & $-0.1386058(10)$ \\
$t_{j}^{21}$ & $0.1853017(7)$ & $-0.9260081(8)$ & $0.1739158(10)$ & $-0.1457194(11)$ & $0.4604202(11)$ \\
$t_{j}^{22}$ & $-0.5089811(7)$ & $0.2551668(9)$ & $-0.4812227(10)$ & $0.4053862(11)$ & $-0.1289875(12)$ \\
\hline
\end{tabular}

